\title{
Estrategias financieras para la eficiencia de las operaciones en departamento de finanzas
}

Financial strategies for the efficiency of operations in the finance department

\begin{tabular}{rrrr}
\cline { 2 - 4 } & José Aguilar & Alfonso León & Dayana Núñez \\
\hline $\begin{array}{r}\text { jose_am1993@hotmail.com } \\
\text { ORCID: 0000-0003-0216-9929 }\end{array}$ & alhd13@gmail.com & dayana08andrea@gmail.com \\
& ORCID: 0000-0003-3748-254 & ORCID: 0000-0003-0688-4743 \\
& Universidad de Carabobo, Venezuela & \\
\hline
\end{tabular}

Artículo recibido en mayo 2019 / Arbitrado en junio 2019 / Publicado en septiembre 2019

RESUMEN $\mid$ El presente estudio tiene como propósito conocer, identificar y evaluar los procedimientos y estrategias financieras aplicadas en el departamento de finanzas de la empresa Febeca, C.A. El estudio se enmarcó dentro de un diseño metodológico no experimental transaccional, del tipo de investigación de campo, con un nivel descriptivo. Se pudo concluir que la empresa objeto de estudio carecía de un proceso de planificación financiera sólido y enfoques orientados a la eficiencia de las operaciones y capacidades internas y externas, para una buena administración y ejecución de sus estrategias financieras dirigidas a la sostenibilidad y crecimiento, lo cual la coloca en situación de riesgo frente a las condiciones políticas, sociales y económicas del país. Partiendo de esta perspectiva se recomendaron una serie de acciones para el desarrollo de las estrategias y garantizar la eficiencia, sostenibilidad y crecimiento de la empresa.

Palabras clave: Estrategias Financieras, sostenibilidad

\begin{abstract}
The purpose of this study is to know, identify and evaluate the procedures and financial strategies applied in the finance department of the company Febeca, C.A. The study was framed within a non-experimental transactional methodological design, of the field research type, with a descriptive level. It was concluded that the company under study lacked a solid financial planning process and approaches oriented to the efficiency of operations and internal and external capacities, for a good administration and execution of its financial strategies aimed at sustainability and growth, which places it at risk from the political, social and economic conditions of the country. Based on this perspective, a series of actions were recommended for the development of strategies and guarantee the efficiency, sustainability and growth of the company.
\end{abstract}

Keywords: Financial Strategies, sustainability 
INTRODUCCIÓN

A nivel mundial las empresas presentan problemáticas comunes en el departamento de finanzas, entre ellas se puede destacar la falta de previsión de pagos, la falta de recursos para la gestión y la falta de comunicación entre departamentos, es así como estos problemas, pueden distorsionar la visión real de los saldos contables que poseen, y conducir a la empresa a deudas, perdidas constantes y retrasos en los pagos a proveedores.

Para Brealey, R, Myers, S y Allen, F (2010) el administrador financiero, o administrador de tesorería enfrenta dos problemas principales:

El primero es decidir cuánto efectivo necesita la empresa en caja y, por lo tanto, cuánto puede invertir en valores que generen intereses. El segundo es asegurarse de que los pagos se realizan con eficiencia. Este administrador no querrá atiborrar su escritorio con los cheques que van llegando hasta que pueda enrollarlos y llevárselos rodando hasta el banco; quiere poner ese dinero en la cuenta bancaria tan pronto como sea posible. (pág.819)

Esto muestra que, hasta los errores más comunes presentados en las organizaciones, pueden causar una mala gestión y disminuir el flujo de efectivo de la empresa provocando una mala imagen delante de los proveedores y clientes, esto último considerado como uno de los activos más preciados de cualquier empresa, su imagen corporativa.

Las estrategias implementadas para el buen funcionamiento y control oportuno en el departamento de finanzas son la columna fundamental del desarrollo de cualquier empresa, ya que este departamento tiene la tarea de hacer un buen manejo de los recursos y tiene como responsabilidad vital brindar seguridad razonable, es decir, presentación fidedigna de las transacciones, ofrecer confianza en los procesos implementados y en la información suministrada a terceros $\sin$ distorsiones materiales, así como la búsqueda continua de mejoras que permitan sobrevivir en medio de las crisis que las entidades comerciales están presentando. Por lo tanto, la mejora en los procesos es una necesidad es cada vez mayor.

Dentro de este marco, las empresas comercializadoras de Venezuela poseen dificultades en el área financiera y al no existir una gestión eficiente ni una adecuada aplicación del control interno dentro de ellas, no se podrá garantizar la liquidez suficiente para poder operar. Por otra parte, los problemas económicos como la inflación e inestabilidad del mercado dentro de la economía nacional también proporcionan un ambiente cambiante para las empresas pertenecientes al sector terciario, el cual es fundamental en la economía de un país.

Siendo así, la empresa Febeca, C.A. comercializadora, perteneciente al sector terciario y clasificada según las Normas Internacionales de Información Financiera (NIIF) como Pequeñas y Medianas Entidades (PYMES), cuenta con cincuenta y cinco (55) años de servicio apostando por el desarrollo de Venezuela, pero en los últimos tiempos ha presentado retrasos en sus procesos operativos por falta de estrategias eficientes capaces de adecuarse a la situación país, generando falta de recursos financieros para la gestión, falta de previsión 
de pagos y en algunas circunstancias mala comunicación entre los departamentos de la empresa.

De esta manera, se realizó una exploración preliminar en el departamento de finanzas y en conversaciones llevadas a cabo con sus colaboradores directos, se pudo conocer que el mismo ha venido presentando los siguientes problemas operativos: Incumplimiento en los plazos para la presentación de la información financiera al departamento de contabilidad, falta de planeación financiera a mediano y largo plazo, retrasos en pagos a proveedores, fallos en los fondos monetarios disponibles para pagos en cuentas bancarias específicas de la empresa, tardanza en la entrega de la información solicitada por las entidades bancarias, demora en la presentación de reportes para la gerencia; en efecto estos problemas son ocasionados por la falta de estrategias financieras eficientes que guíen las actividades dentro del departamento.

Finalmente, se considera la necesidad de profundizar en el tema, ya que también se pudo conocer que no se ha sido realizado un estudio profundo y detallado acerca de cómo pudieran estar influyendo estos problemas en la operatividad y resultados en la gestión financiera de la empresa, y de continuar esta situación puede implicar consecuencias y situaciones negativas 0 desprestigiantes para la imagen corporativa de la entidad con relación a terceros como clientes, proveedores, accionistas, reguladores públicos, auditores externos, empresas filiales, recordando que una buena reputación es clave para futuras relaciones comerciales. Por lo tanto, el objetivo de este estudio es Proponer estrategias financieras con el fin de garantizar la eficiencia de las operaciones en el departamento de finanzas de la empresa Febeca, C.A.

Para tal fin, se considera necesario Describir los problemas que afectan las operaciones dentro del departamento de finanzas de la empresa Febeca, C.A.; Preparar una matriz FODA como base analítica para el departamento de finanzas de la empresa Febeca, C.A.; Analizar las debilidades y fortalezas del departamento de finanzas de la Empresa Febeca, C.A. y finalmente Diseñar estrategias financieras que garanticen la eficiencia dentro del departamento de finanzas de la empresa Febeca, C.A.

Por lo tanto, esta investigación toma como centro de estudio las problemáticas comunes existentes en las empresas comercializadoras, específicamente en sus departamentos de finanzas, para ello es necesario profundizar el estudio de la planeación financiera y la planeación estratégica; por consiguiente, contribuir a detectar y superar las debilidades que se presentan en los mismos.

Por consiguiente, este trabajo busca proporcionar apoyo desde el punto de vista estratégico financiero a las entidades comerciales que se sitúen dentro de la clasificación de las PYME, lo cual aumenta el valor agregado del mismo, dando así una visión más clara acerca del desempeño de las operaciones dentro de los departamentos de Finanzas.

\section{MATERIALES Y METODO}

En el compendio del presente marco metodológico se detallan la forma en la cual se ha realizado el estudio para dar respuesta al problema planteado al inicio de la investigación. 
De acuerdo a las características que presenta este trabajo de investigación, tiene un enfoque mixto, cualitativo y cuantitativo. Cualitativo porque se orienta hacia los factores que influyen en la toma de decisiones para la determinación de las estrategias financieras. Cuantitativo porque para obtener la información pertinente al estudio será necesario realizar y analizar encuestas y comparar los datos obtenidos. El diseño metodológico es el de una investigación de campo no experimental, ya que la investigación se realizó, sin que el investigador manipule ninguna variable.

Así mismo, de acuerdo a las características que presentó el tipo de investigación es de campo debido y su nivel descriptivo; por basarse en la recolección de datos, tomados directamente en el sitio de estudio, conducentes a la obtención de información específica para llegar a las conclusiones y recomendaciones pertinentes.

Para poder desarrollar el estudio es necesario escoger un grupo de individuos de la población establecida los cuales están directamente involucrados con el problema planteado. La población de esta investigación está conformada por los trabajadores del departamento de finanzas en la empresa Febeca, C.A. quienes por ser parte de este departamento en específico poseen características comunes y su número es de diez (10).

En la presente investigación no hay muestra, ya que se consideró la totalidad de la población como objeto de estudio, no se hizo selección de muestra por ser de tipo censal. Con base en eso, se llevará a cabo el estudio tomando a los diez (10) trabajadores pertenecientes al departamento de finanzas de la empresa Febeca.

\section{Técnica e Instrumentos de recolección de datos}

Para esta investigación las técnicas e instrumentos seleccionados son la encuesta, la cual se aplicará a través de un cuestionario de preguntas estructuradas y la técnica de la observación documental o bibliográfica.

Adicionalmente, se empleó la revisión bibliográfica, debido a que forma parte integral de toda investigación, a objeto de facilitar el desarrollo y comprensión del sistema. Bavaresco A, (2013: p.99), considera que "es casi imposible que un estudio escrito carezca de soporte documental, pues conviene siempre revisar lo que ha ocurrido o acontecido en diferentes lugares y tiempo, tanto con las mismas variables o con diferentes; de estudios de reputados autores, artículos científicos o experimentos inéditos". El instrumento que se utilizó para la aplicación de esta técnica fueron los registros anecdóticos.

\section{RESULTADOS Y DISCUSION}

A continuación, se presentan los resultados obtenidos de la aplicación de las técnicas e instrumentos de recolección de datos. Estos resultados, son los que permiten conocer y evaluar los elementos objeto de estudio.

De acuerdo al estudio, existen factores externos no controlables por el empresario que influyen directamente en las finanzas de las organizaciones, como son los, clientes, los proveedores, condiciones macroeconómicas del país, el entorno político, debilidad del mercado interno, alza de precios, inflación, tipo de cambio, entre otros. En este sentido, solo se identificaron que los factores incidentes en el proceso de 
planificación financiera lo constituyen las políticas relacionadas con las leyes gubernamentales.

Vale la pena decir que los factores externos influyen en la forma de reaccionar de los consumidores, así como también, los cambios y las alteraciones que se originen en el entorno pueden afectar los ingresos, los precios y la necesidad de compra del consumidor, esto conlleva a que el crecimiento empresarial sea cada vez menor.

En cuanto al proceso de planificación financiera de estas empresas, el no incorporar mecanismos de medición de resultados que permitan controlar la ejecución de las acciones diseñadas para el logro de objetivos y metas de la gestión financiera, lleva a la empresa a un estado de supervivencia, lo cual cambia si se tuviesen estrategias claras, lo que permitiría un crecimiento quizás no acelerado, pero si constante en una economía tan cambiante como la que posee Venezuela.

En cuanto a la formulación de estrategias, hay ausencia de planes alternativos como una herramienta de control a ser aplicada en la ejecución estratégica de la planificación financiera, con la finalidad de replantear las acciones para corregir la presencia de algunas deficiencias.
Para finalizar, de acuerdo a los resultados de este diagnóstico se puede decir, que el proceso de planificación financiera se realiza de manera deficiente debido a que no se planifican los resultados financieros esperados, existe ausencia de preparación de planes y objetivos estratégicos a corto, mediano y largo plazo, como instrumentos para establecer los posibles escenarios económicos de la organización; En tal sentido se puede catalogar como una gestión reactiva, que actúa de acuerdo a los acontecimientos ocurridos sin anticiparse a ellos, pues no consideran los obstáculos en su entorno.

\section{Matriz FODA}

Del análisis realizado, a través de la aplicación de los instrumentos se pudo conocer información relevante acerca de la problemática planteada, de manos de los actores directos del proceso, lo que facilitó el proceso de generación de estrategias financieras que puedan optimizar los procesos. Los datos obtenidos permitieron la realización de la Matriz FODA, con la intención de describir las debilidades y visualizar las oportunidades que permitan ofrecer soluciones a la problemática planteada. 
Tabla 1. Matriz FODA

\section{Debilidades}

Oportunidades

- No se realiza planificación estratégica financiera

- No existen políticas claras sobre los controles financieros a aplicar

- No existen procedimientos documentados acerca de las estrategias utilizadas en el Departamento.

- Desconocimiento sobre cuáles y qué estrategias se aplican en el Departamento.

- Desconocimiento claro sobre los objetivos y misión que se plantea el Departamento.

- Establecimiento de planes y controles que beneficien la gestión del Departamento.

- Mayor ponderación del Departamento dentro de la toma decisiones de la organización.

- Mejores resultados en la gestión

- Implementación de estrategias apropiadas

\begin{tabular}{lll}
\hline \multicolumn{1}{c}{ Fortalezas } & \multicolumn{1}{c}{ Amenazas } \\
\hline - Constancia, valor corporativo con mucha fuerza & Entrada en vigencia de nuevas medidas \\
en la organización. & & económicas. \\
- Recurso Humano preparado en el área & - Inestabilidad económica. \\
- Reconocimiento de la empresa en su mercado & - Desequilibrio socioeconómico. \\
(entorno) & - Contracción del mercado. \\
\hline
\end{tabular}

Fuente: Aguilar, León y Nuñez (2017)

\section{La Propuesta}

\section{Presentación de la Propuesta}

La presente propuesta que se origina como consecuencia de interpretación y análisis de los resultados obtenidos a través de la aplicación de la encuesta, dirigida a Febeca, C.A. ubicada en la zona Industrial Castillito, Estado Carabobo.

\section{Justificación de la Propuesta}

De acuerdo a los resultados de estudio, la propuesta presentada en esta investigación tiene su justificación en la necesidad de desarrollar una herramienta que cubra la exigencia de garantizar la eficiencia en la ejecución de las operaciones en la empresa Febeca, C.A., elevar la calidad del desempeño en el departamento de finanzas, y aplicar estrategias que proporcionaran beneficios en los procesos diarios.

De esta manera la aplicación de las estrategias financieras apoyadas con la disposición de mejora de los colaboradores de esta entidad, aportara valor en cuanto al rendimiento del departamento, desarrollara el potencial de cada trabajador involucrado en esta propuesta y brindara estrategias solidas que sirvan como base para enfrentar posibles retos financieros. Igualmente permitirá aumentar los niveles de productividad laboral, por ende, contribuye significativamente al logro de la misión y visión de la empresa. A su vez se inyecta en la organización un espíritu de colaboración, una actitud permisiva para analizar $y$ enfrentar los problemas, así como de tener una visión global que permita coordinar y controlar las tareas de manera eficiente.

\section{Objetivos de la Propuesta}

\section{Objetivo General de la Propuesta}

Diseñar estrategias financieras con el fin de asegurar la eficiencia de las operaciones en el departamento de finanzas de la empresa Febeca, C.A. 


\section{Objetivos Específicos de la Propuesta}

- Conocer las actividades requeridas para llevar a cabo las estrategias financieras en el departamento de finanzas.

- Especificar las etapas necesarias para poner en práctica las estrategias financieras en el departamento de finanzas.

- Monitorear las estrategias establecidas dentro del departamento de finanzas.

\section{Factibilidad de la Propuesta}

Factibilidad Institucional: la puesta en marcha de la propuesta, desde el punto de vista institucional no presenta inconveniente, debido que cuenta con la disposición por parte de sus trabajadores como de la gerencia de la empresa.

Factibilidad Operativa: la propuesta es operativa factible, ya que la empresa posee el capital humano capacitado para enfrentar cambios, dispuesto a dar lo mejor de sí para afianzar la eficiencia en sus operaciones, solo se requiere dar a conocer la propuesta en cuento al manejo de las estrategias, y que se tomen en cuenta los procesos que se necesiten.

Factibilidad Técnica: desde el punto de vista técnico, la propuesta es factible, ya que la empresa Febeca, C.A. no necesita realizar inversión monetaria en cuanto a equipos técnicos. Los equipos que se requieren ya están a disposición de los colaboradores y están completamente operativos, como lo es la computadora, laptops, video beam, entre otros.

\section{Alcance de la Propuesta}

El alcance de esta propuesta va dirigido al departamento de finanzas de la empresa Febeca, C.A ubicada en la zona industrial castillito Estado Carabobo municipio San Diego, conformada por diez empleados. Se busca diseñar estrategias financieras que ayuden a mejorar los procesos a fin de contribuir con la visión y misión de la entidad para obtener mejores resultados y corregir los posibles errores que se presentan.

\section{Desarrollo de la Propuesta}

En relación al desarrollo de los objetivos específicos de esta propuesta, los mismos pueden ser alcanzados llevando a cabo las siguientes estrategias financieras en función de lograr la eficiencia en las operaciones del departamento de Finanzas en la empresa Febeca, C.A. Algunas de las estrategias financieras que la organización puede adoptar para maximizar su eficiencia son:

Respecto al Objetivo número uno "Conocer las actividades requeridas para llevar a cabo las estrategias financieras en el departamento de finanzas".

- Realizar un programa de capacitación a los colaboradores del departamento de finanzas en materia de estrategias para poder enfrentar las necesidades de la entidad, que cuente con los conocimientos y la experiencia necesaria para poder resolver las diferentes contingencias que se lleguen a presentar, ante la cambiante situación económica local y nacional. 
- Designar un equipo de trabajo que se encargue de realizar evaluaciones sobre los indicadores financieros, flujos de efectivo y estados financieros, que velen por la adecuada presentación de los saldos reales en bancos en los estados financieros al corte.

- Coordinar actividades que permitan obtener la información necesaria para crear un diagnóstico inicial de la situación financiera de la entidad.

Considerando el estudio antes citado en esta organización realizado Morales, J. y Morales, A. (2014) en cuanto a la planificación financiera estratégica se proponen las siguientes etapas a fin de cubrir el objetivo número dos "Especificar las etapas necesarias para poner en práctica las estrategias financieras en el departamento de finanzas".

Etapa 1. La identificación de problemas y oportunidades: Evaluar el entorno externo (nuevas políticas cambiarias, inestabilidad económica, desequilibrio socio-económico, contracción del mercado y otros aspectos en materia económica y legal) y el ambiente interno (fortalezas y debilidades) del departamento de finanzas. Esta etapa es la del diagnóstico donde se propone implementar una matriz FODA de forma sistemática que permita visualizar aquellos aspectos que afectan el desarrollo de las actividades en el departamento.

Etapa 2. Fijación de metas y objetivos: Entendiendo que la organización está en constante cambio es necesario que se afiancen los objetivos ya establecidos y conocidos por el equipo, bajo los cuales trabajara la empresa, estos deben ser viables y cuantificables.

Los objetivos financieros actúan como guías y son susceptibles a ser modificados para adaptarse a estos posibles cambios. Es por ello que se propone establecer nuevos objetivos en el departamento de finanzas si es necesario, para que cubran los siguientes aspectos:

- Rendimiento sobre la inversión.

- Índice de endeudamiento total (apalancamiento financiero).

- Relación del pasivo a plazo mayor de un año respecto al activo fijo.

- Tasa de interés sobre créditos, entre otros.

Etapa 3. Diseño de un procedimiento para hallar posibles soluciones o caminos para encontrar una solución y escoger la mejor solución: En esta etapa se seleccionan las mejores herramientas que sean factibles para cubrir los objetivos. Se propone como herramienta fundamental la implantación de estrategias financieras incorporadas en otras entidades y que hayan arrojado resultados positivos.

Etapa 4. Procedimientos de control para comprobar los resultados de la solución escogida mediante la planeación estratégica: Una vez que se han cubierto las tres primeras etapas, es necesario estar en constante monitoreo de las estrategias ya establecidas para determinar si los resultados que se van obteniendo son los acertados.

Sobre la base de la consideración anterior radica la importancia del objetivo número tres de esta propuesta el "Monitorear las estrategias establecidas 
dentro del departamento de finanzas." En este mismo sentido se propone afianzar dentro de la organización la evaluación continua de las estrategias financieras ya establecidas, considerando que el entorno socio-económico del país es cambiante y las necesidades son diferentes para cada contexto, por esto se propone:

- Realizar evaluaciones continuas sobre opciones de financiamiento a fin de mantener la inversión en la entidad, para cubrir los presupuestos y metas establecidas.

- Realizar de manera periódica un análisis de liquidez monetaria, de las obligaciones bancarias, y de las posibles herramientas que se puedan utilizar entre los departamentos involucrados en las finanzas de la empresa, como el departamento de cuentas por cobrar y el departamento de cuentas por pagar a fin de medir la situación economía de manera constante.

- Analizar periódicamente los estados financieros para poder tomar adecuadas decisiones.

- Establecer reuniones mensuales entre los colaboradores del departamento de finanzas a fin de actualizarse en materia económica y legal que afecte a la organización.

- Designar un encargado del equipo de finanzas que se encargue de brindar informar de los últimos cambios en materia cambiaria, financiera, legal y de impuestos vía correo electrónica (correo corporativo).

A su vez se proponen las siguientes estrategias que serán de utilidad para el departamento y complementan de manera significativa la propuesta antes expuesta:

- Comparar los presupuestos existentes con los resultados reales para detectar posibles desviaciones y el adecuado uso del efectivo.

- Analizar los manuales de sistemas y procedimientos para asegurarse si las estrategias ya implementadas van en concordancia con las políticas de la entidad.

- Elaborar estudios que muestren gráficamente los resultados obtenidos por el departamento en un periodo determinado.

\section{CONCLUSIONES}

Luego de analizar e interpretar los resultados obtenidos en la aplicación de los instrumentos de recolección de datos y en función a la propuesta referente a: Estrategias financieras que garanticen la eficiencia de las operaciones en el Departamento de Finanzas de la empresa Febeca, C.A., se llegó a las siguientes conclusiones:

1. En su mayoría el personal encuestado del departamento de Finanzas de la Empresa Febeca, C.A. afirman que no existe una planeación financiera dentro del Departamento de finanzas.

2. Según el resultado de la encuesta en el departamento de Finanzas no existe una política clara del establecimiento de nuevos controles o el uso de los controles existentes.

3. La empresa Febeca, C.A. no posee estrategias financieras bien definidas 
para el mejoramiento del proceso operativo del departamento de finanzas.

4. El personal encuestado afirma que la gestión en el departamento de finanzas no es $100 \%$ eficiente, sin embargo, manifestaron que se alcanzan los objetivos, aunque no en el tiempo más adecuado.

5. Existe deficiencia en las operaciones del departamento de finanzas por falta de estrategias financieras.

De acuerdo a lo indicado en el párrafo anterior se puede afirmar que la deficiencia en las operaciones del departamento de finanzas se debe a la falta de herramientas financieras, por ende, en dicho departamento se plantea establecer nuevas estrategias asociadas al crecimiento y la toma de decisiones para el cumplimiento de los objetivos.

Es importante señalar que existen retrasos en las operaciones del departamento de finanzas, debido a que los lineamientos existentes no están bien definidos y no son del conocimiento de todo el personal que labora en dicho departamento. De igual manera el objetivo y la misión del departamento no son conocidos por los integrantes del mismo, por ende, esto ocasiona un impacto perjudicial para la ejecución de las distintas actividades, operativas financieras.

\section{REFERENCIAS}

Arias, F. (2012). El proyecto de investigación. Introducción a la metodología científica. Sexta Edición. Caracas. Editorial Episteme
Baveresco, A. (2013). Proceso Metodológico en la Investigación. Como hacer un Diseño de Investigación. 6a edición. Imprenta internacional, Maracaibo, Venezuela

Bisquerra, R. (2010). Metodología de la Investigación Educativa. Editorial La Muralla, S.A. Madrid, España

Bracho, M. (2012). Gestión financiera de empresas fabricantes de vasos plásticos desechables ubicados en la región zuliana. Maestría en Gerencia Financiera de Empresas Financieras de la Universidad del Zulia

Brealey, R, Myers, S y Allen, F (2010). Principios de Finanzas Corporativas. España. Novena Edición. McGraw Hill

Camacho, A. (2015). Estrategias Financieras a la Industria del Sector Ronero en Venezuela, para el Fortalecimiento de los Costes de las Materias Primas Caso: C. A. Ron Santa Teresa. Trabajo de Grado de la Universidad de Carabobo, Maracay, Edo. Aragua

Calderón, T. (30 de diciembre de 2014). Universo, Población y Muestra. Disponible en: es.slideshare.net: http://es slideshare.net/TomsCaldern/universopoblación-y muestra Consulta 2016, Diciembre 05

Constitución de la República Bolivariana de Venezuela (1999) Publicada en Gaceta Oficial del jueves 30 de diciembre de 1999, N 36.860

Corral, Y. (2014) Instrumentos de recolección de datos: validez y confiabilidad: Para investigaciones de enfoque cualitativo y de enfoque cuantitativo. Editorial Académica Española, Venezuela Edo. Carabobo

Correa, J. (abril de 2010). El Método DOFA, un Método muy Utilizado para Diagnóstico de Vulnerabilidad y Planeación Estratégica. Disponible en: http://www.culturarecreacionydeporte.g ov.co consultado: 15 de noviembre 2016 
Coulter, M. y Robbins, S. (2010) Administración. Décima Edición, Editorial Pearson Educación, México, Consulta: 16 septiembre de 2017

Fundación del Comité de Normas Internacionales de Contabilidad (IASCF) (2009) Norma Internacional de Información Financiera para Pequeñas y Medianas Entidades (NIIF para las PYMES) London, United Kingdom

Gitman, L. (2012). Principios de Administración Financiera. Editorial Pearson Educación. Decimosegunda edición. México

Hernández, R., Fernández, C., \& Baptista, P. (2010). Metodología de la Investigación. México. Editorial: Mc Graw-Hill Education

Koonts, $\mathrm{H}$. Weihrich, $\mathrm{H}$ y Cannice, $\mathrm{M}$. (2012). Administración Una Perspectiva Global y Empresarial. 14ª edición México Editorial: Mc Graw Hill Interamericana Editores S.A

La Reforma del Decreto con Rango, Valor y Fuerza de Ley para la Promoción y Desarrollo de la Pequeña y Mediana Industria y Unidades de Propiedad Social. Decreto $N^{\circ} 1.413$ de fecha 13 de noviembre de 2014

Lobo, K. (2014) Estrategia Gerencial para la Optimización de la Gestión Administrativa de la Empresa Servicios Todito 2020, C.A. Caracas, Venezuela. Agosto 2014

Morales, J. y Morales, A. (2014) Planeación Financiera. Primera Edición, Grupo Editorial Patria, S.A. México, DF

Nuñez, J. (2014). Estrategia Financiera Para La Pequeña Y Mediana Empresa PYME. Disponible en https://www.gestiopolis.com/estrategiafinanciera-para-la-pequena-y-medianaempresa-pyme/ Consultado: 05 de diciembre de 2016

Palella, S. y Martins, F. (2012). Metodología de la investigación cuantitativa. 3a Edición 1aㅡ Reimpresión Caracas. FEDUPEL, Fondo Editorial de la Universidad Pedagógica Experimental Libertador

Romero, E. (2011) Un marco de referencia para garantizar un control interno adecuado en una PYME industrial argentina. Trabajo de grado en línea. Universidad Tecnológica de Buenos Aires. Documento en línea Disponible: http://www.posgrado.frba.utn. edu.ar/investigación/tesis /MAN-2011Rom.pd Consulta: octubre 2016

Sabino, C. (2014) El Proceso de la Investigación. Editorial Episteme. Caracas-Venezuela

Salvatierra, M. (2010), "Estrategias financieras bajo la perspectiva del cuadro de mando integral para el crecimiento sostenible de punto salud Valencia C.A. Trabajo de Grado de Magister en Administración de Empresas Mención Finanzas. Universidad de Carabobo. Valencia. Venezuela

Serna, H. (2011) Gerencia Estratégica. Teoría-Metodología-Alineamiento, Implementación y Mapas Estratégicos, Índices de Gestión. 11므 edición. Editorial: 3R Editores. Bogotá, Colombia

Tamayo, M. (2011). El Proceso de la Investigación Científica. Editorial Limusa Noriega Editores, México, Ciudad de México, Quinta Edición

Universidad de Carabobo (2011). Normativa para los Trabajos de investigación de la facultada de ciencias económicas y sociales de la Universidad de Carabobo. Publicaciones Faces UC, Bárbula, Venezuela

Wheelen, T. y Hunger, J. (2010) Administración Estratégica y Política de Negocios. Conceptos y Casos. Decima Edición, Editorial Pearson Educación, México 